\title{
Influence of Postponed Follow-Up after Cochlear Implant Activation during the COVID-19 Pandemic on Aided Sound Field Detection and Speech Recognition
}

\author{
Teresa G. Vos ${ }^{a}$ Kevin D. Brown ${ }^{a}$ Emily Buss ${ }^{a}$ Andrea L. Bucker ${ }^{b}$ \\ Matthew M. Dedmon ${ }^{\mathrm{a}}$ Brendan P. O'Connell ${ }^{\mathrm{a}}$ Jenna Raymond ${ }^{\mathrm{b}}$ \\ Margaret T. Dillon ${ }^{a}$ \\ aDepartment of Otolaryngology/Head \& Neck Surgery, University of North Carolina at Chapel Hill, Chapel Hill, NC, \\ USA; 'Department of Audiology, UNC Health Care, Chapel Hill, NC, USA
}

\section{Keywords}

Electric threshold - Mapping • Electric-acoustic stimulation ·

Clinical protocol

\begin{abstract}
Introduction: The objective of this study was to assess the influence of postponing the first post-activation follow-up due to the COVID-19 pandemic on the aided sound field detection thresholds and speech recognition of cochlear implant $(\mathrm{Cl})$ users. Methods: A retrospective review was performed at a tertiary referral center. Two groups of adult $\mathrm{Cl}$ recipients were evaluated: (1) patients whose first post-activation follow-up was postponed due to COVID-19 closures (postponed group; $n=10$ ) and (2) a control group that attended recommended post-activation follow-ups prior to the COVID-19 pandemic (control group; $n=18$ ). For both groups, electric thresholds were estimated at initial activation based on comfort levels and were measured behaviorally at subsequent post-activation follow-ups. For the control group, behavioral thresholds were measured at the 1-month follow-up. For the postponed group, behavioral thresholds were not measured until 3 months post-activation since the 1-month follow-up was postponed. The aided pure-tone average (PTA) and word recognition results were
\end{abstract}

karger@karger.com

(c) 2021 S. Karger AG, Basel

www.karger.com/aud

Karger! compared between groups at the 3-month follow-up and at an interim visit 2-9 weeks later. Results: At the 3-month follow-up, the postponed group had significantly poorer word recognition ( 23 vs. $42 \%, p=0.027$ ) and aided PTA (42 vs. 37 $\mathrm{dB} \mathrm{HL}, p=0.041$ ) than the control group. No significant differences were observed between 3-month data from the control group and interim data from the postponed group. Conclusions: The postponed follow-up after $\mathrm{Cl}$ activation was associated with poorer outcomes, both in terms of speech recognition and aided audibility. However, these detrimental effects were reversed following provision of an individualized map, with behaviorally measured electric threshold and comfort levels. While adult $\mathrm{Cl}$ recipients demonstrate an improvement in speech recognition with estimated electric thresholds, the present results suggest that behavioral mapping within the initial weeks of device use may support optimal outcomes.

(c) 2021 S. Karger AG, Basel

\section{Introduction}

The management of cochlear implant (CI) recipients includes a series of postoperative follow-up encounters for medical evaluation, device activation, mapping, and 
performance assessment. According to the 2019 American Academy of Audiology Clinical Practice Guidelines, the recommended follow-ups within the first year of implantation for adult CI recipients include device activation approximately $1-4$ weeks postoperatively and then 1 week, 1 month, 3 months, 6 months, and 12 months postactivation [Messersmith et al., 2019]. CI centers vary in their recommended follow-ups, although the majority of centers recommend that $\mathrm{CI}$ recipients are seen within the first few weeks after device activation to support optimal speech recognition [Vaerenberg et al., 2014].

Evaluation of CI recipient performance at post-activation follow-ups includes assessment of aided detection and speech recognition with the device. The CI recipient may listen with either electric stimulation alone (CI alone) or the combination of ipsilateral electric-acoustic stimulation (EAS), depending on the residual acoustic hearing in the implanted ear. First, aided sound field detection and speech recognition with familiar map settings are assessed. Aided detection thresholds indicate the softest sounds the listener can perceive across speech frequency range (e.g., 125-8,000 kHz) with the device, which are influenced by the electric thresholds [Vargas et al., 2012]. Speech recognition is evaluated with recorded materials from the Minimum Speech Test Battery [MSTB, 2011] to assess performance as compared to preoperative abilities and previous visits. Post-lingually deafened adult CI recipients typically experience significant improvements in speech recognition during the first 6 months of device use and subsequently plateau [Lenarz et al., 2012].

After evaluation of patient performance, mapping procedures are completed to individualize device settings. Routine mapping procedures for CI-alone and EAS devices include the determination of: (1) electrical thresholds, or the minimum stimulation level, and (2) the upper stimulation level. For adult CI recipients, these levels are typically obtained through behavioral responses [Vargas et al., 2012]. Objective measures like the electrically evoked stapedial reflex threshold (ESRT) can be used to estimate the upper stimulation levels [Jerger et al., 1988; Brickley et al., 2005]. For some cases, assigning the upper stimulation levels based on the ESRT is more efficient than behavioral measures; however, ESRT is not observed in all CI recipients [Hodges et al., 1997].

Also, obtaining an accurate behavioral measurement of electric threshold levels at device activation is challenging, given the lack of listening experience [Vargas et al., 2012]. The clinical mapping software for CI devices includes algorithms to estimate the electric threshold levels. For instance, MED-EL software allows the assignment of electric threshold using a percentage (e.g., 10\% of the associated upper stimulation level). Audiologists may choose to estimate electric threshold levels at device activation and measure electric thresholds at a subsequent encounter to individualize the map. The need for behaviorally measured electric thresholds is unclear as some CI recipients have demonstrated similar speech recognition when listening with a map using estimated or interpolated electric threshold levels as compared to a map using behaviorally measured electric threshold levels [Plant et al., 2005; Spahr and Dorman, 2005; Shapiro and Bradham, 2012; Vargas et al., 2012; Rader et al., 2018]. The clinical protocol at our institution for devices utilizing CIS-type signal coding is to use clinical software algorithms to assign electric threshold levels at device activation and measure electric thresholds behaviorally on all active channels at post-activation follow-ups.

Fluctuations in the upper stimulation levels with initial device use have been reported and support the measurement of electric thresholds and upper stimulation levels on all channels in the early post-activation period [Skinner, 2003]. Previous investigations have demonstrated variable time periods when electric threshold and upper stimulation levels stabilize, ranging from 3 to 12 months post-activation [Brown et al., 2000; Franck and Norton, 2001; Hughes et al., 2001; Henkin et al., 2003; Vargas et al., 2012]. For example, Vargas et al. [2012] have noted a mean increase in upper stimulation levels of $54 \%$ and decrease in electric threshold levels of $22 \%$ between device activation and 6 months post-activation, and approximately stable values afterward. These changes are thought to be due to acclimatization to electrical stimulation and increased tolerance for higher stimulation levels [Hughes et al., 2001], including reactivation of the auditory pathway and cortex with neuron recruitment and reduction of neuronal activation thresholds [Vargas et al., 2012]. Progressive increases in impedances associated with postoperative intra-cochlear fibrosis may also play a role in the progressive increase in upper stimulation levels [Vargas et al., 2012]. Consequently, the clinical protocol for post-activation follow-ups includes aided sound field assessments and individualized mapping on all active channels.

In the setting of the COVID-19 pandemic in early 2020 , most CI centers limited in-person encounters to cases identified as "critical" to reduce clinician and patient volume in the clinic. At our institution, critical visits for adult patients were defined as device activations or suspected device failures. Subsequently, there was a period between March 2020 and May 2020 when CI recipi- 
ents were not seen for routine post-activation follow-ups. Some CI recipients were not seen between device activation and the 3-month follow-up, that is, they missed the 1-month follow-up. The present study investigated the influence of missing the 1-month post-activation followup on aided sound field detection thresholds and speech recognition for adult CI recipients. The first aim was to compare aided sound field detection thresholds and speech recognition at the 3-month follow-up between CI recipients who were mapped at the 1-month follow-up (control group; visit completed prior to the COVID-19 pandemic) and CI recipients who missed the 1-month follow-up due to the initial response to the COVID-19 pandemic (postponed group). The second aim was to assess whether the performance of the postponed group at a later interim visit converged with the performance of the control group at the 3-month follow-up.

\section{Methods}

The retrospective review of the speech recognition and map settings for CI recipients was approved by the University of North Carolina at Chapel Hill IRB (IRB protocol 09-2328). The adult CI database was queried to obtain demographic information, aided sound field detection thresholds, aided speech recognition performance, and map settings.

\section{Subjects}

The postponed group included adult CI recipients ( $>18$ years of age at implantation) who underwent cochlear implantation and initial device activation at the study site between December 2019 and March 2020, missed their 1-month post-activation follow-up due to the COVID-19 pandemic, and returned for their 3-month followup. The control group comprised CI recipients who underwent cochlear implantation and initial device activation at the study site between April 2019 and November 2019, and attended the recommended follow-ups (i.e., 1-month and 3-months post-activation). Exclusion criteria included revision surgery, history of acoustic neuroma, or inner-ear malformation on the implanted side. The database query included recipients of a single manufacturer (i.e., MED$\mathrm{EL} \mathrm{GmbH)}$ to increase homogeneity between groups.

\section{Procedures}

The clinical protocol included assessment of aided sound field detection thresholds and speech recognition. Patients were seated in a soundproof booth, facing a speaker at $0^{\circ}$ azimuth approximately $1 \mathrm{~m}$ away. At the preoperative visit, aided detection and recognition measures were completed with patients listening with an appropriately-fit hearing aid on the ear-to-be-implanted. At the post-activation follow-ups, patients listened with their familiar map. For cases with substantial hearing in the contralateral ear, masking was presented via an insert earphone. Aided sound field detection thresholds were assessed with pulsed warble tones from 125 to $8,000 \mathrm{~Hz}$ using an ascending approach. Patients responded by either pressing a response button or raising their hand. The aided speech recognition test battery included the Consonant-Nucleus-Consonant (CNC) monosyllabic words test [Peterson and Lehiste, 1962]. Recorded materials were presented at $60 \mathrm{~dB}$ SPL. Patients were instructed to repeat the word they heard and encouraged to guess if unsure. Performance was scored as the percent of words correctly repeated.

Mapping of CI-alone and EAS devices was conducted after the assessment of aided detection thresholds and speech recognition with MED-EL MAESTRO software (version 8.0.3). All patients were mapped with the FS4 coding strategy, with sensitivity set at $75 \%$. The upper stimulation level was assigned as the most comfortable loudness (MCL) level, which is defined as the highest stimulation level a patient can listen to comfortably for a sustained period of time [Shapiro and Bradham, 2012; Vaerenberg et al., 2014]. An ascending approach was used to behaviorally measure the MCL level for each channel. Patients indicated the perceived loudness on a provided graphic loudness ranking scale. The stimulation level for an individual channel was increased until the patient indicated the perceived loudness to be "uncomfortably loud." The MCL level for each channel was assigned at the highest stimulation level that the patient indicated the loudness to be "loud, but comfortable." At device activation, the primary goal was to obtain sufficient audibility with the device to support improved speech recognition and to counsel on external components. Thus, the clinical protocol at the study site for MED-EL recipients was to estimate electric thresholds at $10 \%$ of the behaviorally measured MCL levels. At the post-activation follow-ups, electric thresholds and MCL levels were measured behaviorally on all active channels. The electric threshold levels are programmed at a level that is 2 units below the softest level that the patient detects the signal 100\% of the time. At the early intervals (e.g., $<6$ months), patients are provided with 3 additional maps with progressively increased MCL levels. Procedures for mapping the electric component are the same for EAS users as for CI-alone users, with the exception of the assignment of the electric frequency filters [Incerti et al., 2013]. The acoustic component for EAS users is fit to NAL-NL1 prescriptive targets at device activation [Byrne et al., 2001; Dillon et al., 2014].

\section{Data Analysis}

The primary aim was to investigate whether missing the 1-month follow-up influenced aided detection thresholds and speech recognition after 3 months of device use. A rationalized arcsine transform was applied to the percent correct data prior to analysis to normalize error variance [Studebaker, 1985]. An independent samples two-tailed $t$-test compared the CNC score obtained at the 3-month follow-up between the postponed and control groups, using SPSS (version 26). An aided pure-tone average (PTA) was calculated by averaging the aided sound field detection thresholds at 500, 1,000, and 2,000 Hz. An independent samples 2-tailed $t$-test compared the aided PTA at the 3-month follow-up between the postponed and control groups. A bivariate Pearson correlation assessed the relationship between aided PTA and the CNC score.

The second aim was to assess whether the speech recognition of the postponed group at an interim visit (2-9 weeks after 3-month follow-up) converged with the performance of the control group. An independent samples 2-tailed $t$-test compared the CNC scores of the postponed group at the interim visit to the scores of the control group at the 3-month follow-up. For all analyses, significance was defined as $\propto<0.05$. 
Table 1. Demographic data for the control and postponed groups

\begin{tabular}{|c|c|c|}
\hline & Control group $(n=18)$ & Postponed group $(n=10)$ \\
\hline \multirow[t]{2}{*}{ Age at implantation, years } & Range: 52-84 & Range: $40-85$ \\
\hline & Mean: 67 (SD 10.7) & Mean: 67 (SD 14.8) \\
\hline Gender, $n(\%)$ & Female: 7 (39) & Female: 4 (40) \\
\hline \multirow[t]{2}{*}{ Duration of deafness, years } & Range: 0-75 & Range: $1-33$ \\
\hline & Mean: 30 (SD 22) & Mean: 17 (SD 10) \\
\hline \multirow[t]{2}{*}{ Preoperative PTA, dB HL } & Range: $57-118$ & Range: $58-98$ \\
\hline & Mean: 71 (SD 25) & Mean: 80 (SD 14) \\
\hline \multirow[t]{2}{*}{ Preoperative aided CNC, \% correct } & Range: $0-56$ & Range: $0-52$ \\
\hline & Mean: 16 (SD 17) & Mean: 14 (SD 18) \\
\hline \multirow[t]{3}{*}{ Electrode array, $n(\%)$} & Flex24: $3(17)$ & Flex24: $2(20)$ \\
\hline & Flex28: $2(11)$ & Flex28: $2(20)$ \\
\hline & FlexSoft: 13 (72) & FlexSoft: $6(60)$ \\
\hline \multirow[t]{2}{*}{ External processor (Cl alone or EAS), $n(\%)$} & $\mathrm{Cl}$ alone: $14(78)$ & $\mathrm{Cl}$ alone: 7 (70) \\
\hline & EAS: $4(22)$ & EAS: $3(30)$ \\
\hline Second-side Cl (i.e., contralateral), $n$ (\%) & $2(11)$ & $2(20)$ \\
\hline
\end{tabular}

$\mathrm{Cl}$, cochlear implant; EAS, electric-acoustic stimulation; CNC, Consonant-Nucleus-Consonant; PTA, pure-tone average.

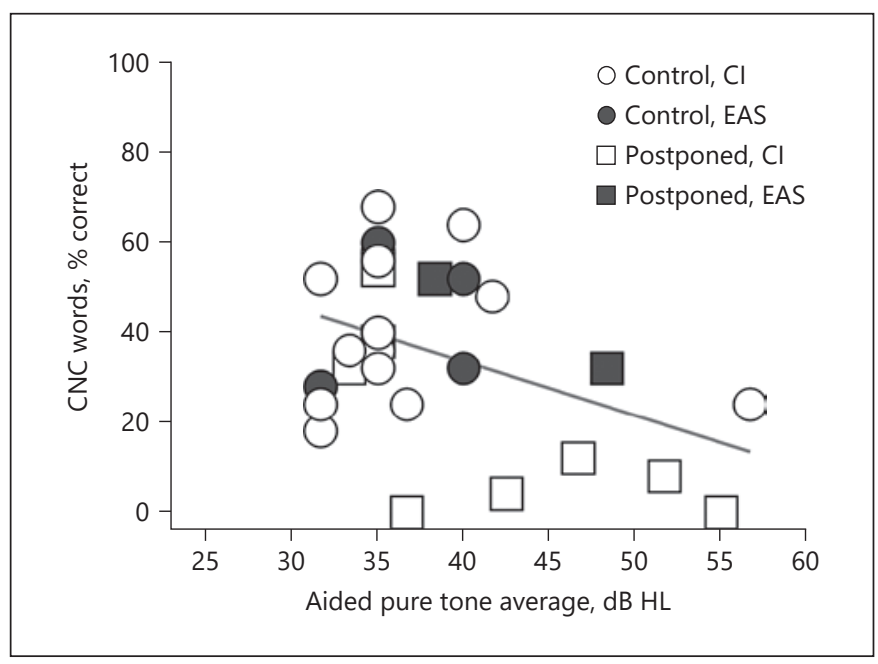

Fig. 1. Aided CNC scores (plotted as percent correct) at the preoperative and post-activation intervals for the postponed and control groups. CI, cochlear implant; EAS, electric-acoustic stimulation; $\mathrm{CNC}$, Consonant-Nucleus-Consonant; PTA, pure-tone average.

\section{Results}

Ten patients ( 4 female) met inclusion criteria for the postponed group, and 18 patients ( 7 female) met criteria for the control group. Demographic information is listed in Table 1. The age at implantation ranged from 40 to 85 (mean: 67; SD: 15) years for the postponed group and from 52 to 84 (mean: 67; SD: 11) years for the control group. The mean preoperative aided CNC score was $14 \%$ (SD: $18 \%$ ) for the postponed group and 16\% (SD: 17\%) for the control group. The groups did not differ significantly for age at implantation $\left(t_{(26)}=0.01, p=0.992\right)$ or preoperative aided CNC scores for the ear-to-be-implanted $\left(t_{(26)}=-0.43, p=0.669\right)$. The distribution of electrode arrays was broadly similar between groups, with the majority being FlexSOFT in both the postponed (60\%) and control (72\%) groups. The distribution of EAS versus CI-alone users was similar between the groups, with EAS users comprising $30 \%$ of the postponed group and $22 \%$ of the control group. All EAS users had electric low-frequency filters $<500 \mathrm{~Hz}$, thus all frequencies included in the PTA fell within the electric filter region for all patients. Impedance for all active electrode contacts was within normal limits for both groups.

Figure 1 plots the CNC scores of the postponed and control groups obtained at the preoperative visit and post-activation follow-ups. Percent correct performance for the control group was represented by hatched boxes, and performance for the postponed group was represented by open boxes. At the 3-month follow-up, there was a significant difference in speech recognition between groups $\left(t_{(11.9)}=-2.52, p=0.027\right)$, with better performance observed for the control group (mean: $42 \%$, SD: $16 \%$ ) than for the postponed group (mean: 23\%, SD: 21\%). However, the postponed group did experience a significant improvement in word recognition at the 3-month 


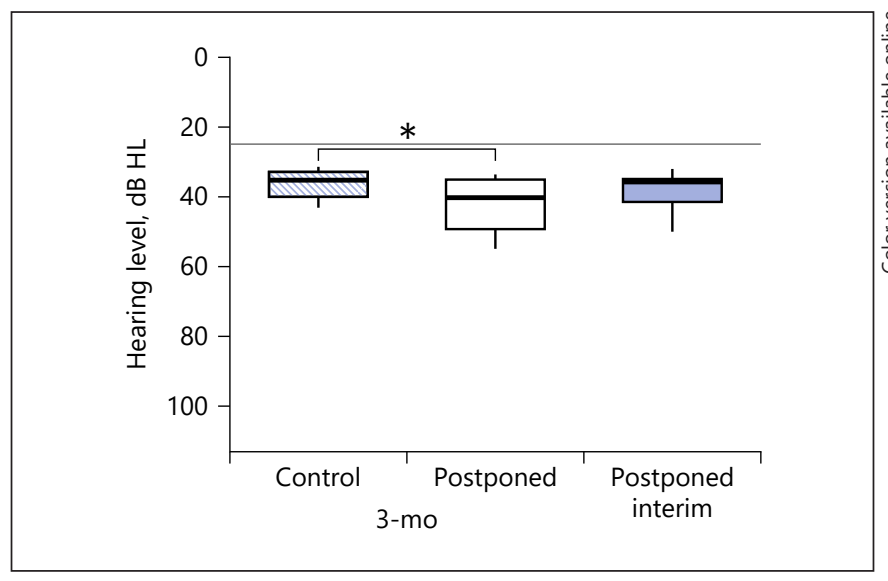

Fig. 2. Aided PTA with the familiar map for the control and postponed groups at the 3-month interval and for the postponed group at the interim visit. The horizontal line indicates the level for normal hearing (i.e., $25 \mathrm{~dB}$ HL). PTA, pure-tone average; mo, months.

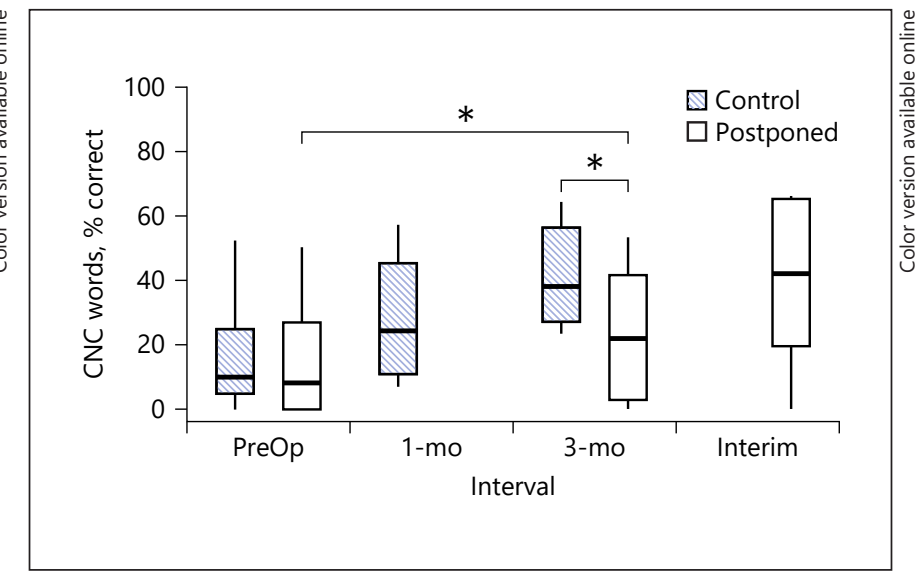

Fig. 3. Association between aided PTA and CNC scores (plotted as percent correct) at the 3-month follow-up. The legend indicates the specific group and device for each individual. CNC, Consonant-Nucleus-Consonant; PTA, pure-tone average. follow-up compared to preoperative scores (23\% correct compared to $14 \%$ preoperatively, $t_{(9)}=-2.89, p=0.018$ ).

Figure 2 plots the aided PTA; results are shown separately for the control and the postponed groups at the 3-month follow-up, and for the postponed group at the interim visit. The aided PTA at the 3-month follow-up was significantly higher for the postponed group than the control group (mean $42 \mathrm{~dB}$ HL vs. $37 \mathrm{~dB}$ HL; $t_{(26)}=-2.15$, $p=0.041)$. However, there was overlap in values between the groups (33-55 dB HL and 28-57 dB HL, respectively). Figure 3 plots the association between aided PTA and CNC scores at the 3-month follow-up for individuals in the control (circles) and postponed (squares) groups. Open symbols indicate CI-alone users and filled symbols indicate EAS users. There was a significant negative correlation between aided PTA and $\mathrm{CNC}$ scores at the 3 -month follow-up $\left(r_{(28)}=-0.45, p=0.016\right)$, indicating that patients who could detect stimuli at softer presentation levels demonstrated better speech recognition.

The second aim was to assess whether the performance of the postponed group after the 3-month follow-up converged with the performance of the control group. Behavioral mapping of both electric threshold and MCL levels was first completed for the postponed group at the 3 -month follow-up. Eight of the 10 patients from the postponed group returned approximately 2-9 weeks after the 3-month follow-up for an interim visit. At the interim visit, the aided PTA ranged from 35 to $50 \mathrm{~dB} \mathrm{HL}$ (mean: $38 \mathrm{~dB}$ HL; SD: $6 \mathrm{~dB} \mathrm{HL}$ ), and CNC scores ranged from 0 to $66 \%$ (mean: $40 \%$; SD: $25 \%$ ). Comparisons of these re- sults to those of the control group at the 3-month followup found no significant difference for aided PTA $\left(t_{(13.6)}=\right.$ $0.67, p=0.514)$ or CNC scores $\left(t_{(8.6)}=-0.42, p=0.688\right)$. This is consistent with the conclusion that the performance of the postponed group converged with the performance observed for the control group after completing individualized mapping.

\section{Discussion}

Routine mapping within the initial months after device activation accounts for changes in electric threshold and upper stimulation levels, and supports better speech recognition for CI recipients [Brown et al., 2000; Franck and Norton, 2001; Hughes et al., 2001; Henkin et al., 2003; Lenarz et al., 2012; Vargas et al., 2012]. In this study, adult CI recipients who missed their initial post-activation encounter (i.e., 1-month follow-up) secondary to limited clinic visits during the COVID-19 pandemic demonstrated significantly poorer performance at the 3-month follow-up than a sample of patients who returned for the 1-month follow-up and underwent mapping with behaviorally measured electric thresholds and MCL levels prior to the pandemic. The benefit of individualized mapping of electric thresholds and MCL levels at the 1-month follow-up was further supported by the finding that the performance of most patients in the postponed group converged with the performance of the control group after individualized electric threshold and MCL level mapping 
procedures. The present findings support the recommendation for CI recipients to return for assessment and mapping within the initial weeks post-activation to support optimal speech recognition with their CI-alone or EAS device.

At the 3-month follow-up, the postponed group was listening with maps based on estimated electric thresholds (i.e., $10 \%$ of MCL level for a given channel), and the control group was listening with maps based on behaviorally measured electric thresholds. The study findings suggest that behaviorally measured electric thresholds may support greater audibility of speech information by providing detection of speech information at softer levels [Firszt et al., 2004], in contrast with studies that have found no significant difference between speech recognition with maps based on interpolated or estimated electric thresholds as compared to behaviorally measured electric thresholds [Plant et al., 2005; Spahr and Dorman, 2005; Boyd, 2006]. Typically, in the initial months after device activation, the upper stimulation levels increase [Hughes et al., 2001; Henkin et al., 2003; Vargas et al., 2012] and the electric thresholds decrease [Vargas et al., 2012]. Extended listening with maps created at initial activation may result in poorer performance since the upper stimulation levels may be too low and the estimated threshold levels may be imprecise. Considering patients were provided with progressively louder maps (i.e., globally increased MCL levels), the outcome differences observed in the present sample were thought to be due to at least partially to the differences in how the electric threshold levels were assigned (i.e., estimated vs. measured).

The postponed group was unable to return to clinic due to initial scheduling restrictions in response to the COVID-19 pandemic to reduce in-person interactions. While performance was significantly poorer than observed in patients who were able to return for the 1-month follow-up prior to the pandemic, the postponed group did experience a significant improvement in word recognition at the 3-month follow-up compared to preoperative performance. This demonstrates that use of clinical software algorithms to estimate electric thresholds supports map settings that improve speech recognition with the device; however, further improvements in early speech recognition are expected with a map using behaviorally measured electric thresholds.

It is important to note that the study conclusions are limited by the nature of retrospective analysis of data and a small sample size. Duration of daily device use, which has been observed to be positively associated with speech recognition for CI recipients [Holder et al., 2020], was not consistently available in this sample. The sample size did not warrant stratification of performance differences between CI-alone and EAS device users. Users of EAS demonstrate better word recognition in quiet than listening with a CI-alone [Kiefer et al., 2005; Dillon et al., 2015]; however, comparisons of word recognition in quiet for EAS users with short arrays versus CI-alone users with long arrays demonstrate similar performance [Büchner et al., 2017]. In the present sample, EAS users did not demonstrate substantially better CNC scores than CI-alone users (see Fig. 3). Further investigation is needed to determine whether optimal mapping procedures of electric threshold and upper stimulation levels differ for CI-alone versus EAS users. Additionally, it is possible that the control group may have also demonstrated further improvement in speech recognition if evaluated at the interim visit.

While the present results are interpreted as reflecting a benefit of measuring electric threshold levels, we cannot rule out a beneficial effect of remeasuring MCL levels at the first post-activation encounter. Improved performance after post-activation mapping could also be observed even if electric thresholds were estimated based on MCL levels, although this seems unlikely considering electric threshold levels typically decrease, and increases in MCL levels would result in overestimated electric threshold levels. A prospective, randomized investigation is needed to determine whether prolonging the initial follow-up period after device activation negatively influences speech recognition, and if behaviorally measured electric thresholds support better aided sound field detection thresholds and speech recognition in the initial months of device use than estimated electric thresholds. Additionally, only one CI manufacturer was included in this review to control for differences in electrode array design, signal coding, and mapping procedures. The optimal mapping procedures within the initial months of device use may differ for other CI manufacturers.

\section{Conclusions}

The observed improvement in speech recognition after individualized mapping of electric thresholds in adult CI recipients with postponed follow-up is particularly timely in light of modified procedures in response to the COVID-19 pandemic. While many clinics have reopened, some continue to employ modified scheduling procedures, and many patients demonstrate continued hesitancy to attend appointments. It is essential to adhere to 
appropriate protective measures in the face of a pandemic, and medical risks must be weighed by patients and clinicians. Adult CI recipients demonstrate a significant benefit when listening to maps with estimated electric thresholds within the initial months of device use as compared to preoperative performance, although the magnitude of the performance growth may be less than if listening to maps with behaviorally measured electric thresholds. The present findings suggest the need to counsel patients that optimal performance may not be reached when follow-ups are missed, particularly in the early period after device activation. If delays or extensions between follow-ups are recommended to limit exposure in clinic, clinicians should prioritize individualized mapping procedures, such as behavioral measurement of electric thresholds, to support optimal performance. Implementation of mapping via telemedicine may be an alternative, althougheffectiveprocedurestoassessperformance with the device (e.g., aided detection thresholds and speech recognition) would be needed to support optimal mapping.

\section{Acknowledgments}

Sarah Dillon, AuD, Kristen Quinones, AuD, Adrienne Pearson, $\mathrm{AuD}$, Noelle Roth, AuD, and Allison Young, AuD conducted aided detection and recognition assessments and mapping for the study sample.

\section{Statement of Ethics}

This study protocol was approved by the University of North Carolina IRB (IRB protocol 09-2328). According to the IRB protocol, informed written consent was waived in the standard fashion for studies involving no more than minimal risk to subjects without additional research testing required.

\section{Conflict of Interest Statement}

K.D.B. serves on the surgical advisory board for MED-EL Corporation. B.P.O. is a consultant for Advanced Bionics Corporation, Johnson \& Johnson, and MED-EL Corporation. A.L.B. serves on the audiology advisory board for MED-EL Corporation. M.T.D. is supported by a research grant from MED-EL Corporation provided to the university.

\section{Funding Sources}

M.T.D. is supported by a research grant from MED-EL Corporation provided to the university; no funding was directly provided to this study.

\section{Author Contributions}

Teresa G. Vos contributed to data acquisition and analysis, and wrote the manuscript. Kevin D. Brown contributed to interpretation of data and critical revisions of the manuscript. Emily Buss contributed to study design, data analysis, and critical revisions of the manuscript. Andrea L. Bucker contributed to acquisition of data and critical revisions of the manuscript. Matthew M. Dedmon contributed to interpretation of data and critical revisions of the manuscript. Brendan P. O'Connell contributed to interpretation of data and critical revisions of the manuscript. Jenna Raymond contributed to acquisition of data and critical revisions of the manuscript. Margaret T. Dillon contributed to study design, data analysis, and critical revisions of the manuscript.

\section{Data Availability Statement}

All data generated or analyzed during this study are included in this article. Further inquiries can be directed to the corresponding author.

\section{References}

Brickley G, Boyd P, Wyllie F, O’Driscoll M, Webster D, Nopp P. Investigations into electrically evoked stapedius reflex measures and subjective loudness percepts in the MED-EL COMBI $40+$ cochlear implant. Cochlear Implants Int. 2005;6(1):31-42.

Brown CJ, Hughes ML, Luk B, Abbas PJ, Wolaver A, Gervais J. The relationship between EAP and EABR thresholds and levels used to program the nucleus 24 speech processor: data from adults. Ear Hear. 2000;21(2):151-63.

Boyd PJ. Effects of programming threshold and maplaw settings on acoustic thresholds and speech discrimination with the MED-EL
COMBI $40+$ cochlear implant. Ear Hear. 2006;27(6):608-18.

Büchner A, Illg A, Majdani O, Lenarz T. Investigation of the effect of cochlear implant electrode length on speech comprehension in quiet and noise compared with the results with users of electro-acoustic-stimulation, a retrospective analysis. PLoS One. 2017;12(5): e0174900.

Byrne D, Dillon H, Ching T, Katsch R, Keidser G. NAL-NL1 procedure for fitting nonlinear hearing aids: characteristics and comparisons with other procedures. J Am Acad Audiol. 2001;12(1):37-51.
Dillon MT, Buss E, Pillsbury HC, Adunka OF, Buchman CA, Adunka MC. Effects of hearing aid settings for electric-acoustic stimulation. J Am Acad Audiol. 2014;25(2):133-40.

Dillon MT, Buss E, Adunka OF, Buchman CA, Pillsbury HC. Influence of test condition on speech perception with electric-acoustic stimulation. Am J Audiol. 2015;24(4):520-8.

Firszt JB, Holden LK, Skinner MW, Tobey EA, Peterson A, Gaggl W, et al. Recognition of speech presented at soft to loud levels by adult cochlear implant recipients of three cochlear implant systems. Ear Hear. 2004;25(4):37587. 
Franck KH, Norton SJ. Estimation of psychophysical levels using the electrically evoked compound action potential measured with the neural response telemetry capabilities of cochlear corporation's CI24M device. Ear Hear. 2001;22(4):289-99.

Henkin Y, Kaplan-Neeman R, Muchnik C, Kronenberg J, Hildesheimer M. Changes over time in electrical stimulation levels and electrode impedance values in children using the nucleus $24 \mathrm{M}$ cochlear implant. Int J Pediatr Otorhinolaryngol. 2003;67(8):873-80.

Hodges AV, Balkany TJ, Ruth RA, Lambert PR, Dolan-Ash S, Schloffman JJ. Electrical middle ear muscle reflex: use in cochlear implant programming. Otolaryngol Head Neck Surg. 1997;117(3 Pt 1):255-61.

Holder JT, Dwyer NC, Gifford RH. Duration of processor use per day is significantly correlated with speech recognition abilities in adults with cochlear implants. Otol Neurotol. 2020;41(2):e227-31.

Hughes ML, Vander Werff KR, Brown CJ, Abbas PJ, Kelsay DM, Teagle HF, et al. A Longitudinal Study of electrode impedance, the electrically evoked compound action potential, and behavioral measures in nucleus 24 cochlear implant users. Ear Hear. 2001;22(6):471-86.
Incerti PV, Ching TY, Cowan R. A systematic review of electric-acoustic stimulation: device fitting ranges, outcomes, and clinical fitting practices. Trends Amplif. 2013;17(1):3-26.

Jerger J, Oliver TA, Chmiel RA. Prediction of dynamic range from stapedius reflex in cochlear implant patients. Ear Hear. 1988;9(1):4-8.

Kiefer J, Pok M, Adunka O, Stürzebecher E, Baumgartner W, Schmidt M, et al. Combined electric and acoustic stimulation of the auditory system: results of a Clinical Study. Audiol Neurootol. 2005;10(3):134-44.

Lenarz M, Sönmez H, Joseph G, Büchner A, Lena$\mathrm{rz} \mathrm{T}$. Long-term performance of cochlear implants in postlingually deafened adults. Otolaryngol Head Neck Surg. 2012;147(1):112-8.

Messersmith JJ, Entwisle L, Warren S, Scott M. Clinical practice guidelines: cochlear implants. J Am Acad Audiol. 2019;30(10):82744.

MSTB: The New Minimum Speech Test Battery. http: //www.auditorypotential.com/MSTB_ Nav.html. Published online 2011.

Peterson GE, Lehiste I. Revised CNC lists for auditory tests. J Speech Hear Disord. 1962;27: 62-70.

Plant K, Law MA, Whitford L, Knight M, Tari S, Leigh J, et al. Evaluation of streamlined programming procedures for the nucleus cochlear implant with the contour electrode array. Ear Hear. 2005;26(6):651-68.
Rader T, Doms P, Adel Y, Weissgerber T, Strieth S, Baumann U. A method for determining precise electrical hearing thresholds in cochlear implant users. Int J Audiol. 2018;57(7): 502-9.

Shapiro WH, Bradham TS. Cochlear implant programming. Otolaryngol Clin North Am. 2012;45(1):111-27.

Skinner MW. Optimizing cochlear implant speech performance. Ann Otol Rhinol Laryngol Suppl. 2003;191:4-13.

Spahr AJ, Dorman MF. Effects of minimum stimulation settings for the Med El Tempo+ speech processor on speech understanding. Ear Hear. 2005;26(4 Suppl):2S-6S.

Studebaker GA. A "rationalized" arcsine transform. J Speech Lang Hear Res. 1985;28(3): $455-62$.

Vaerenberg B, Smits C, De Ceulaer G, Zir E, Harman S, Jaspers N, et al. Cochlear implant programming: a global survey on the state of the art. Sci World J. 2014;2014:501738-12.

Vargas JL, Sainz M, Roldan C, Alvarez I, de la Torre A. Long-term evolution of the electrical stimulation levels for cochlear implant patients. Clin Exp Otorhinolaryngol. 2012;5(4): 194-200. 